\title{
EXISTENCE, UNIQUENESS, AND REGULARITY \\ OF SOLUTIONS TO SEMILINEAR RETARDED DIFFERENTIAL EQUATIONS
}

D. BAHUGUNA

Received 12 October 2003 and in revised form 22 May 2004

In the present work, we consider a semilinear retarded differential equation in a Banach space. We first establish the existence and uniqueness of a mild solution and then prove its regularity under different additional conditions. Finally, we consider some applications of the abstract results.

\section{Introduction}

Consider the following semilinear retarded differential equation in a Banach space $X$ :

$$
\begin{gathered}
u^{\prime}(t)+A u(t)=f\left(t, u_{t}\right), \quad t \in(0, T], \\
h\left(u_{0}\right)=\phi,
\end{gathered}
$$

where $0<T<\infty ; \phi \in \mathscr{C}_{0}:=C([-\tau, 0] ; X), \tau>0$; the linear operator $A$, defined from the domain $D(A) \subset X$ into $X$, is such that $-A$ generates a $C_{0}$ semigroup $\{S(t): t \geq 0\}$ of bounded linear operators in $X$; the nonlinear map $f$ is defined from $[0, T] \times \mathscr{C}_{T}, \mathscr{C}_{T}:=$ $C([-\tau, T] ; X)$, into $X$; the map $h$ is defined from $\mathscr{C}_{0}$ into $\mathscr{C}_{0}$; and for $(t, u) \in[0, T] \times \mathscr{C}_{T}$, the function $u_{t} \in \mathscr{C}_{0}$ is given by $u_{t}(s)=u(t+s)$ for $s \in[-\tau, 0]$. Here $\mathscr{C}_{t}:=C([-\tau, t] ; X)$ for $t \in[0, T]$ is the Banach space of all continuous functions from $[-\tau, t]$ into $X$ endowed with the supremum norm

$$
\|\phi\|_{t}=\sup _{-\tau \leq s \leq t}\|\phi(s)\|, \quad \phi \in \mathscr{C}_{t},
$$

where $\|\cdot\|$ is the norm in $X$.

The existence and uniqueness results for (1.1) may also be applied to the particular cases, namely, the semilinear retarded functional differential equation

$$
\begin{gathered}
u^{\prime}(t)+A u(t)=f\left(t, u_{t}\right), \quad t \in(0, T], \\
u_{0}=\phi,
\end{gathered}
$$


and a retarded differential equation with a nonlocal history condition

$$
\begin{gathered}
u^{\prime}(t)+A u(t)=f\left(t, u_{t}\right), \quad t \in(0, T], \\
g\left(u_{0}\right)=x,
\end{gathered}
$$

where $g: \mathscr{C}_{0} \rightarrow X$, and $x \in X$. In this case, we may take $h(\psi)(s) \equiv g(\psi)$ and $\phi(s) \equiv x$ for $s \in[-\tau, 0]$.

Presently, we will be more concerned with the applications of the results for the case (1.4), though we present the analysis here for the more general case (1.1). The linear case of (1.3), in which $f(t, \phi)=L \phi$ with a bounded linear operator $L: \mathscr{C}_{T} \rightarrow X$, is recently considered by Bátkai et al. [3] using the theory of perturbed Hille-Yosida operators.

The theory of functional differential equations with the history conditions of the type considered in (1.1) and (1.4) may be applied to the epidemic population dynamic models. For such related works, we refer to Alaoui [1] and references cited therein.

For the earlier works on existence, uniqueness, and stability of various types of solutions of differential and functional differential equations with nonlocal conditions, we refer to Byszewski and Akca [5], Byszewski and Lakshmikantham [6], Byszewski [4], Balachandran and Chandrasekaran [2], Lin and Liu [7], and references cited in these papers.

We note that any $\psi \in \mathscr{C}_{T_{2}}$ also belongs to $\mathscr{C}_{T_{1}}, 0<T_{1} \leq T_{2} \leq T$, as the restriction of $\psi$ on the subinterval $\left[-\tau, T_{1}\right]$ of $\left[-\tau, T_{2}\right]$. Let $\tilde{T}$ be any number such that $0<\tilde{T} \leq T$. We define a map $F: \mathscr{C}_{\tilde{T}} \rightarrow \mathscr{C}_{\tilde{T}}$ by

$$
(F \psi)(t)= \begin{cases}\psi(t), & t \in[-\tau, 0], \\ S(t) \psi(0)+\int_{0}^{t} S(t-s) f\left(s, \psi_{s}\right) d s, & t \in[0, \tilde{T}] .\end{cases}
$$

We assume that there exists $\chi \in \mathscr{C}_{T}$ such that $h\left(\chi_{0}\right)=\phi$. Let $\mathscr{W}(\chi, \tilde{T}):=\left\{\psi \in \mathscr{C}_{\tilde{T}}: \psi_{0}=\chi_{0}\right\}$. The set $\mathscr{W}(\chi, \tilde{T})$ consists of functions in $\mathscr{C}_{\tilde{T}}$, those which coincide with the function $\chi$ on $[-\tau, 0]$. Clearly, for $\psi \in \mathcal{W}(\chi, \tilde{T}), F \psi \in \mathcal{W}(\chi, \tilde{T})$. A function $u \in \mathcal{W}(\chi, \tilde{T})$ such that $F u=u$ on $[-\tau, \tilde{T}]$ is called a mild solution of $(1.1)$ on $[-\tau, \tilde{T}]$. By a strong solution $u$ of (1.1) on $[-\tau, \tilde{T}]$, we mean a function $u \in \mathscr{C}_{\tilde{T}}$ such that $u$ is differentiable a.e. on $[0, \tilde{T}]$, $u(t) \in D(A)$ for a.e. $t \in[0, \tilde{T}], h\left(u_{0}\right)=\phi$, and

$$
u^{\prime}(t)+A u(t)=f\left(t, u_{t}\right), \quad \text { a.e. } t \in(0, \tilde{T}]
$$

By a classical solution $u$ of $(1.1)$ on $[-\tau, \tilde{T}]$, we mean a function $u \in \mathscr{C}_{\tilde{T}}$ such that $u \in$ $C^{1}((0, \tilde{T}] ; X), u(t) \in D(A)$ for $t \in(0, \tilde{T}]$, and $u$ satisfies $(1.1)$ on $[-\tau, \tilde{T}]$. We first prove the existence of a mild solution of $(1.1)$ on $\left[-\tau, t_{0}\right]$ for some $0<t_{0} \leq T$ and then prove that this mild solution can be extended uniquely to a mild solution of (1.1) either on the whole $[-\tau, T]$ or to the maximal interval $\left[-\tau, t_{\max }\right), 0<t_{\max } \leq T$, and since $t_{\max }<\infty$, we obtain

$$
\lim _{t \rightarrow t_{\max }-}\|u(t)\|=+\infty .
$$

We then establish the regularity of this mild solution under different additional assumptions. Finally, we consider some applications of the results obtained. 


\section{Preliminaries and main result}

We assume the following hypotheses.

(A1) The linear operator $A: D(A) \subset X \rightarrow X$ is such that $-A$ is the infinitesimal generator of a $C_{0}$-semigroup $\{S(t): t \geq 0\}$ of bounded linear operators in $X$.

(A2) Suppose that there exists $\chi \in \mathscr{C}_{T}$ such that $h\left(\chi_{0}\right)=\phi$.

(A3) The nonlinear map $f:[0, T] \times \mathscr{C}_{0} \rightarrow X$ satisfies a local Lipschitz-like condition

$$
\|f(t, \psi)-f(s, \tilde{\psi})\| \leq L_{f}(r)\left[|t-s|+\|\psi-\tilde{\psi}\|_{0}\right]
$$

for all $t, s \in[0, T]$ and $\psi, \tilde{\psi} \in B_{R}\left(\mathscr{C}_{0}, \chi_{0}\right)$, where $L_{f}:[0, \infty) \rightarrow[0, \infty)$ is a nondecreasing function and for $r>0$,

$$
B_{r}\left(\mathscr{C}_{0}, \chi_{0}\right)=\left\{\psi \in \mathscr{C}_{0}:\left\|\psi-\chi_{0}\right\| \leq r\right\}
$$

Theorem 2.1. Suppose that the conditions (A1)-(A3) are satisfied. Then (1.1) has a mild solution $u$ either on the whole interval $[-\tau, T]$ or on the maximal interval of existence $\left[-\tau, t_{\max }\right), 0<t_{\max } \leq T$, and in the later case, $\lim _{t \rightarrow t_{\max }-}\|u(t)\|=\infty$. Moreover, if $\chi$ is Lipschitz continuous on $[-\tau, 0]$ and $\chi(0) \in D(A)$, then $u$ is Lipschitz continuous, in the first case, on $[-\tau, T]$, otherwise, in the second case, on every compact subinterval of $\left[\tau, t_{\mathrm{max}}\right)$. If, in addition, $X$ is reflexive, then $u$ is a strong solution of (1.1) on the interval of existence. If, in addition, $S(t)$ is an analytic semigroup in $X$, then $u$ is a classical solution of (1.1) on the interval of existence. The solution $u$ is unique if and only if $\chi$ is unique on $[-\tau, 0]$.

\section{Proof of Theorem 2.1}

We first establish the existence of a mild solution $u$ on $\left[-\tau, t_{0}\right], 0<t_{0} \leq T$, and then prove its unique continuation. For a fixed $R>0$ and any $0<\tilde{T} \leq T$, let

$$
\mathscr{W}(\chi, \tilde{T})_{R}=\left\{\psi \in \mathcal{W}(\chi, \tilde{T}):\|\psi-\chi\|_{\tilde{T}} \leq R\right\} .
$$

Choose $0<t_{0} \leq T$ such that for $0<t \leq t_{0}$, we have

$$
\|(S(t)-I) \chi(0)\| \leq \frac{R}{3}, \quad\|\chi(t)-\chi(0)\| \leq \frac{R}{3}, \quad t_{0} \cdot M_{0} \leq \min \left\{\frac{3}{4}, \frac{R}{3}\right\},
$$

where

$$
M_{0}=M e^{\omega T}\left[L_{f}(R)\left(T+R+2\|\chi\|_{T}\right)+\left\|f\left(0, \chi_{0}\right)\right\|\right]
$$

and the constants $M \geq 1, \omega \geq 0$ are such that

$$
\|S(t)\| \leq M e^{\omega t}, \quad t \geq 0 .
$$

For $\psi \in \mathcal{W}\left(\chi, t_{0}\right)_{R}$, it may be verified easily that $F \psi \in \mathcal{W}\left(\chi, t_{0}\right)_{R}$. Let $\psi_{1}, \psi_{2} \in \mathcal{W}\left(\chi, t_{0}\right)_{R}$. Then

$$
\left\|\left(F \psi_{1}\right)-\left(F \psi_{2}\right)\right\|_{t_{0}} \leq t_{0} \cdot M e^{\omega T} L_{f}(R)\left\|\psi_{1}-\psi_{2}\right\|_{t_{0}} \leq t_{0} M_{0}\left\|\psi_{1}-\psi_{2}\right\|_{t_{0}} .
$$


Since $t_{0} M_{0}<1$, it follows that there exists a unique $u \in \mathcal{W}\left(\chi, t_{0}\right)$ such that $u$ is a mild solution on $\left[-\tau, t_{0}\right]$.

If $t_{0}<T$, consider the problem

$$
\begin{gathered}
w^{\prime}(t)+A w(t)=\tilde{f}\left(t, w_{t}\right), \quad t \in\left(0, T-t_{0}\right], \\
\tilde{h}\left(w_{0}\right):=w_{0}=\tilde{\phi},
\end{gathered}
$$

where

$$
\begin{gathered}
\tilde{f}(t, \psi)=f\left(t+t_{0}, \psi\right), \quad t \in\left[0, T-t_{0}\right], \\
\tilde{\phi}(t)=u\left(t+t_{0}\right), \quad t \in\left[-\tau-t_{0}, 0\right] .
\end{gathered}
$$

Since the functions $\tilde{f}$ satisfy (A3) on translated interval and (A2) is automatically satisfied in this case, we may proceed as before to get a function $w \in C\left(\left[-\tau-t_{0}, T-t_{0}\right] ; X\right)$ such that $w$ is a mild solution of (3.6) on $\left[-\tau-t_{0}, t_{1}\right]$ for some $0<t_{1} \leq T-t_{0}$. This $w$ is unique as $\tilde{h}$ is the identity map. Then

$$
\bar{u}(t)= \begin{cases}u(t), & t \in\left[-\tau, t_{0}\right] \\ w\left(t-t_{0}\right), & t \in\left[t_{0}, t_{0}+t_{1}\right]\end{cases}
$$

is a mild solution of $(1.1)$ on $\left[-\tau, t_{0}+t_{1}\right]$. Continuing this way, we get either a function $u \in \mathscr{C}_{T}$, unique in $\mathscr{W}(\chi, T)$, which is a mild solution of $(1.1)$ on $[-\tau, T]$, or $u \in$ $C\left(\left[-\tau, t_{\max }\right) ; X\right), 0<t_{\max } \leq T$, such that for every sufficiently small $\epsilon>0$, there exists a unique $u_{\epsilon} \in \mathscr{W}(\chi, T-\epsilon)$ with $u=u_{\epsilon}$ on $\left[-\tau, t_{\max }-\epsilon\right]$ and $u_{\epsilon}$ is a mild solution of (1.1) on $\left[-\tau, t_{\max }-\epsilon\right]$. In the later case, (1.7) holds; otherwise, we may extend $u$ beyond $t_{\max }$ contradicting the definition of the maximal interval of existence $\left[-\tau, t_{\max }\right.$ ) (cf. $[8$, Theorem 1.4, pages 185-187]).

Now, suppose that $\chi$ is Lipschitz continuous on $[-\tau, 0]$ and $\chi(0) \in D(A)$. We prove that $u$ is Lipschitz continuous on $[-\tau, T]$ for the first case. For the second case when $u$ exists on $\left[-\tau, t_{\max }\right), t_{\max } \leq T$, the proof can be modified by establishing the Lipschitz continuity of $u$ on every compact subinterval $[-\tau, \tilde{T}], \tilde{T}<t_{\max }$. Let $t \in[-\tau, T]$ and $h \geq 0$. If $t+h \leq 0$, then

$$
\|u(t+h)-u(t)\|=\|\chi(t+h)-\chi(t)\| \leq L_{\chi} h
$$

for some positive constant $L_{\chi}$. If $t \leq 0$ and $t+h>0$, then $|t| \leq h$ and $|t+h| \leq h$. In this case, we have

$$
\begin{aligned}
\|u(t+h)-u(t)\| \leq & \|(S(t+h)-I) \chi(0)\|+\|\chi(t)-\chi(0)\| \\
& +\int_{0}^{t+h}\|S(t+h-\eta)\|\left\|f\left(\eta, u_{\eta}\right)\right\| d \eta \\
\leq & C_{1}(R) h
\end{aligned}
$$


for some positive constant $C_{1}(R)$ depending on $R$ only. For the case when $t>0$, we have

$$
\begin{aligned}
\|u(t+h)-u(t)\| \leq & \|(S(h)-I) S(t) \chi(0)\| \\
& +\int_{0}^{t+h}\left\|\left[S(t+h-\eta) f\left(\eta, u_{\eta}\right)-S(t) f\left(\eta, u_{\eta}\right)\right]\right\| d \eta .
\end{aligned}
$$

Replacing $\eta$ by $\eta-h$, we get

$$
\begin{aligned}
\|u(t+h)-u(t)\| \leq & \|(S(h)-I) S(t) \chi(0)\| \\
& +\int_{0}^{t}\left\|S(t-\eta)\left[f\left(\eta+h, u_{\eta+h}\right)-S(t) f\left(\eta, u_{\eta}\right)\right]\right\| d \eta \\
& +\int_{-h}^{0}\left\|S(t-\eta) f\left(\eta+h, u_{\eta+h}\right)\right\| d \eta \\
\leq & C_{2} h+C_{3}(R) \int_{0}^{t}\left\|u(\eta+h)-u_{\eta}\right\| d \eta
\end{aligned}
$$

for some positive constants $C_{2}$ and $C_{3}(R)$. Combining (3.9), (3.10), and (3.12), we have, for $t \in[-\tau, T], h \geq 0$, and $t-\tau \leq \bar{t} \leq t$,

$$
\begin{aligned}
& \|u(\bar{t}+h)-u(\bar{t})\| \\
& \quad \leq A(R) h+B(R) \int_{0}^{t}\left[\sup _{-\tau \leq \theta \leq 0}\|u(\eta+h+\theta)-u(\eta+\theta)\|\right] d \eta
\end{aligned}
$$

for two positive constants $A(R)$ and $B(R)$ depending on $R$ only. Letting $\bar{t}=t+\bar{\theta}, \bar{\theta} \in$ $[-\tau, 0]$, and taking the supremum on $\bar{\theta}$ over $[-\tau, 0]$, we get

$$
\begin{aligned}
\sup _{-\tau \leq \theta \leq 0} & \|u(t+h+\theta)-u(t+\theta)\| \\
& \leq A(R) h+B(R) \int_{0}^{t}\left[\sup _{-\tau \leq \theta \leq 0}\|u(\eta+h+\theta)-u(\eta+\theta)\|\right] d \eta .
\end{aligned}
$$

Application of Gronwall's inequality in (3.14) implies that

$$
\|u(t+h)-u(t)\| \leq \sup _{-\tau \leq \theta \leq 0}\|u(t+h+\theta)-u(t+\theta)\| \leq D(T, R) h
$$

for some positive constant $D(T, R)$ depending on $T$ and $R$ only. Thus, $u$ is Lipschitz continuous on $[-\tau, T]$.

Also, for proving the remaining part of Theorem 2.1, we assume the interval of existence $[-\tau, T]$. The proof may be modified for the second case by replacing the function $u$ and the interval $[-\tau, T]$ with $u_{\epsilon}$ and $\left[-\tau, t_{\max }-\epsilon\right]$, respectively, for every sufficiently small $\epsilon>0$. Now, suppose that $u(0) \in D(A)$ and $X$ is reflexive. The function $\bar{f}:[0, T] \rightarrow X$ given by

$$
\bar{f}(t)=f\left(t, u_{t}\right)
$$


is Lipschitz continuous and therefore differentiable a.e. on $[0, T]$ and $\bar{f}^{\prime}$ is in $L^{1}((0, T) ; X)$. Consider the Cauchy problem

$$
\begin{gathered}
v^{\prime}(t)+A v(t)=\bar{f}(t), \quad t \in(0, T] \\
v(0)=u(0) .
\end{gathered}
$$

By [8, Corollary 2.10, page 109], there exists a unique strong solution $v$ of $(3.17)$ on $[0, T]$. Clearly, $\tilde{v}$ defined by

$$
\tilde{v}(t)= \begin{cases}u(t), & t \in[-\tau, 0], \\ v(t), & t \in[0, T]\end{cases}
$$

is a strong solution of $(1.1)$ on $[-\tau, T]$. But this strong solution is also a mild solution of (1.1) and $\tilde{v} \in \mathcal{W}(\chi, T)$. By the uniqueness of such a function in $\mathcal{W}(\chi, T)$, we get $\tilde{v}(t)=u(t)$ on $[-\tau, T]$. Thus $u$ is a strong solution of (1.1). If $S(t)$ is analytic semigroup in $X$, then we may use [8, Corollary 3.3, page 113] to obtain that $u$ is a classical solution of (1.1). Clearly, if $\chi \in \mathscr{C}_{T}$ satisfying $h\left(\chi_{0}\right)=\phi$ is unique on $[-\tau, 0]$, then $u$ is unique. If there are two $\chi$ and $\tilde{\chi}$ in $\mathscr{C}_{T}$ satisfying $h\left(\chi_{0}\right)=h\left(\tilde{\chi}_{0}\right)=\phi$, with $\chi_{0} \neq \tilde{\chi}_{0}$, then $\mathcal{W}(\chi, T) \cap \mathcal{W}(\tilde{\chi}, T)=\varnothing$ and hence the solutions $u$ and $\tilde{u}$ of (1.1) belonging to $\mathcal{W}(\chi, T)$ and $\mathscr{W}(\tilde{\chi}, T)$, respectively, are different. This completes the proof of Theorem 2.1.

\section{Applications}

Theorem 2.1 may be applied to get the existence, uniqueness, and regularity results for (1.1) in the case when the operator $A$, with the domain $D(A)=W^{2 m, p}(\Omega) \cap W_{0}^{m, p}(\Omega)$ into $X:=L^{p}(\Omega), 1<p<\infty$, is associated with the strongly elliptic partial differential operator

$$
A u=\sum_{|\alpha| \leq 2 m} a_{\alpha}(x) D^{\alpha} u
$$

in a bounded domain $\Omega$ in $\mathbb{R}^{n}$ with sufficiently smooth boundary $\partial \Omega$.

In (1.1) or (1.4), we may take $f$ as the function $f:[0, T] \times C([-\tau, 0] ; X) \rightarrow X$ given by

$$
f(t, \psi)=f_{0}(t)+\int_{-\tau}^{0}\left(\int_{\Omega} \psi(s)(x) d x\right) k_{1}(s) \psi(s) d s, \quad \psi \in C([-\tau, 0] ; X),
$$

where $f_{0}:[0, T] \rightarrow X$ is a Lipschitz continuous function on $[0, T]$, and $k_{1} \in L^{1}(-\tau, 0)$. In (1.4), we consider the following cases of the function $g: \mathscr{C}_{0} \rightarrow X$.

(g1) For $\psi \in \mathscr{C}_{0}$, let

$$
g(\psi)=\int_{-\tau}^{0} k_{2}(s) \psi(s) d s
$$

where $k_{2} \in L^{1}((-\tau, 0))$ with $\kappa=\int_{-\tau}^{0} k_{2}(s) d s \neq 0$. For a given $x \in X$ (we assume $x \in D(A)$ for regularity), we may take $\chi \in \mathscr{C}_{T}$ as $\chi(t) \equiv(1 / \kappa) x, t \in[-\tau, T]$. 
(g2) Let $-\tau \leq a_{1}<a_{2}<\cdots<a_{l} \leq 0$. Let $c_{i} \geq 0$ for $i=1,2, \ldots, l$ and $C:=\sum_{i=1}^{l} c_{i} \neq 0$. For $\psi \in \mathscr{C}_{0}$, let

$$
g(\psi)=\sum_{i=1}^{l} c_{i} \psi\left(a_{i}\right)
$$

For a given $x \in X\left(x \in D(A)\right.$ for regularity), we may take $\chi \in \mathscr{C}_{T}$ as $\chi(t)=(1 / C) x, t \in$ $[-\tau, T]$.

(g3) With $\psi, a_{i}$, and $c_{i}$ as in (g2) and $\epsilon_{i}>0$ for $i=1,2, \ldots, l$, let

$$
g(\psi)=\sum_{i=1}^{l} \frac{c_{i}}{\epsilon_{i}} \int_{a_{i}-\epsilon_{i}}^{a_{i}} u(s) d s .
$$

For any given $x \in X\left(x \in D(A)\right.$ for regularity), $\chi \in \mathscr{C}_{T}$ may be chosen as in (g2).

\section{Acknowledgment}

The author would like to thank the National Board for Higher Mathematics for providing the financial support to carry out this work under its research project no. NBHM/2001/ R\&D-II.

\section{References}

[1] L. Alaoui, Nonlinear homogeneous retarded differential equations and population dynamics via translation semigroups, Semigroup Forum 63 (2001), no. 3, 330-356.

[2] K. Balachandran and M. Chandrasekaran, Existence of solutions of a delay differential equation with nonlocal condition, Indian J. Pure Appl. Math. 27 (1996), no. 5, 443-449.

[3] A. Bátkai, L. Maniar, and A. Rhandi, Regularity properties of perturbed Hille-Yosida operators and retarded differential equations, Semigroup Forum 64 (2002), no. 1, 55-70.

[4] L. Byszewski, Theorems about the existence and uniqueness of solutions of a semilinear evolution nonlocal Cauchy problem, J. Math. Anal. Appl. 162 (1991), no. 2, 494-505.

[5] L. Byszewski and H. Akca, Existence of solutions of a semilinear functional-differential evolution nonlocal problem, Nonlinear Anal. 34 (1998), no. 1, 65-72.

[6] L. Byszewski and V. Lakshmikantham, Theorem about the existence and uniqueness of a solution of a nonlocal abstract Cauchy problem in a Banach space, Appl. Anal. 40 (1991), no. 1, 11-19.

[7] Y. P. Lin and J. H. Liu, Semilinear integrodifferential equations with nonlocal Cauchy problem, Nonlinear Anal. 26 (1996), no. 5, 1023-1033.

[8] A. Pazy, Semigroups of Linear Operators and Applications to Partial Differential Equations, Applied Mathematical Sciences, vol. 44, Springer-Verlag, New York, 1983.

D. Bahuguna: Department of Mathematics, Indian Institute of Technology Kanpur, Kanpur208 016, India

E-mail address: d_bahuguna@yahoo.com 


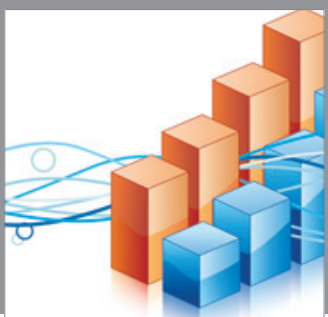

Advances in

Operations Research

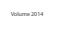

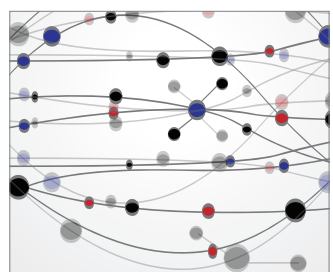

\section{The Scientific} World Journal
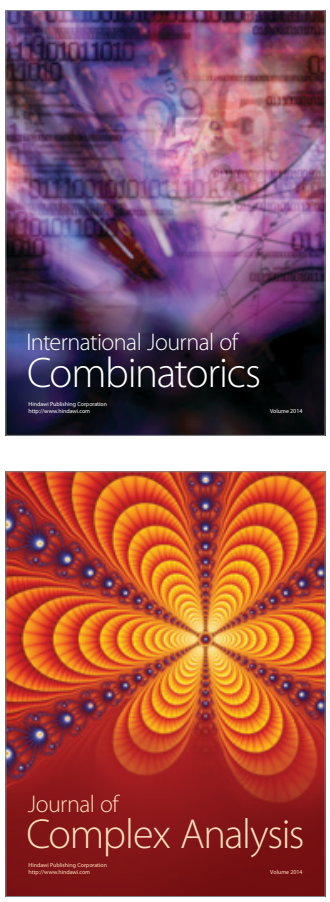

International Journal of

Mathematics and

Mathematical

Sciences
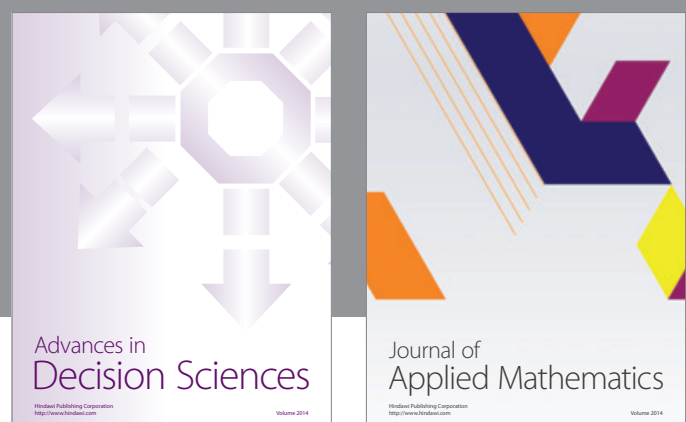

Journal of

Applied Mathematics
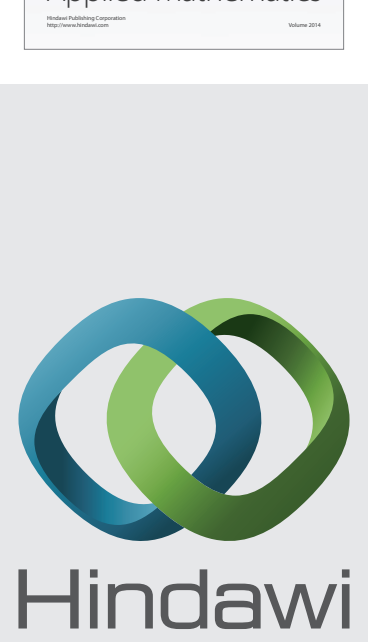

Submit your manuscripts at http://www.hindawi.com
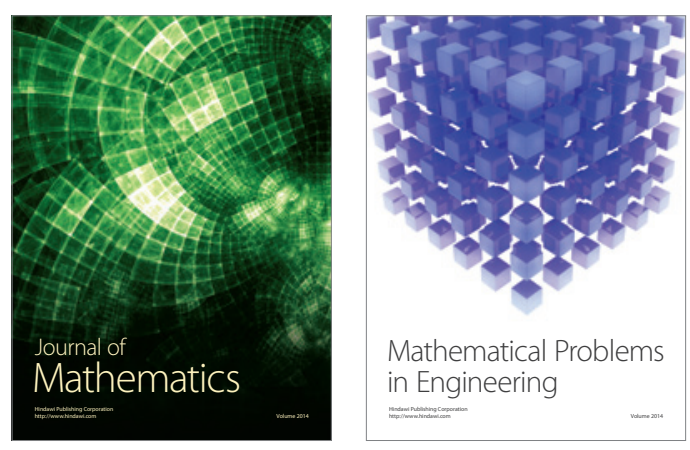

Mathematical Problems in Engineering
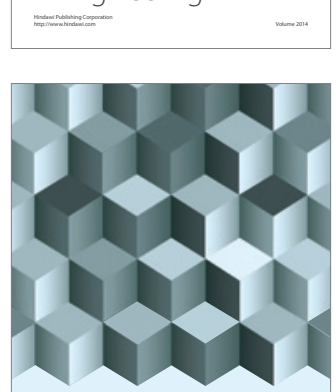

Journal of

Function Spaces
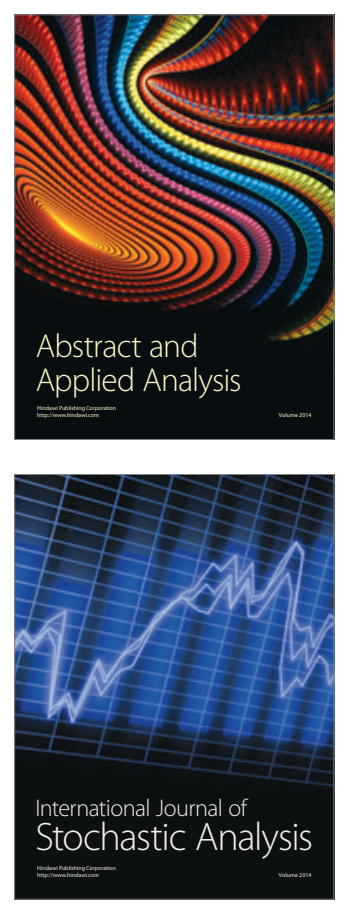

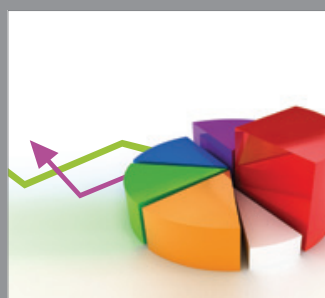

ournal of

Probability and Statistics

Promensencen
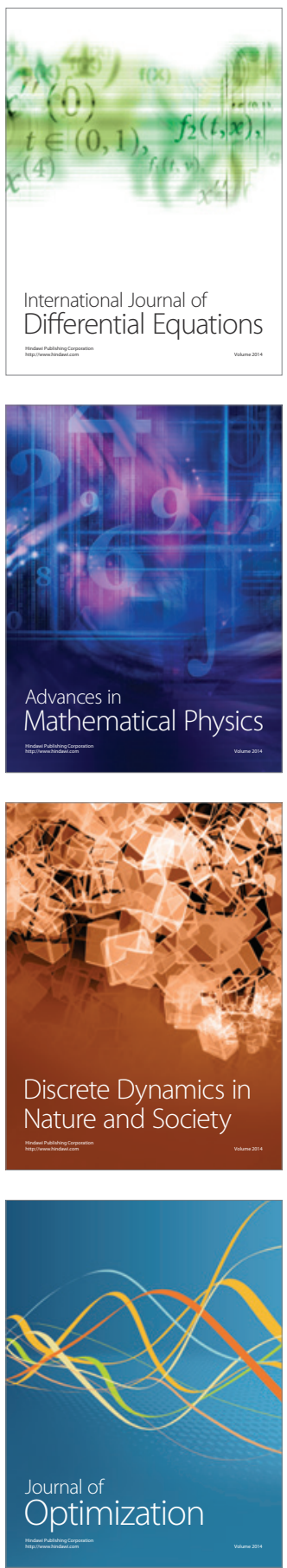